\title{
About the fashion show music
}

\author{
Hai Yan Guo \\ wuhan university of textile clothing school of hubei wuhan 430073 \\ Email:50154681@qq.com
}

Keywords: Fashion show; Music; Rhythm and style

\begin{abstract}
With the prosperity of economy, people's life quality rise ceaselessly, the footsteps of people for the pursuit of beauty also moment without ceasing. The pursuit of beauty can be appreciated fashion show some ways to achieve a goal, such as fashion show is a comprehensive art, and music is a part of the art and music for the performance of the model have the most direct and sentiment, the influence of music is very important in a fashion show, is shown to the clothing and models show mastery, to perfect interpretation of the clothing. Each clothes has its own unique temperament of music, music is necessary to the success of a fashion show, it offers fashion show the visual and auditory enjoy an ideal environment, discuss from the rhythm of music, rhythm, timbre and dress can bring to the audience's emotional resonance, the aesthetic feeling of mining both in form, thus revealing the music sound effect in the fashion show a style of music. Fashion show as the best combination of art beauty, most people have been more recognition and appreciation. Enjoy music in the fashion show, realize music interact with clothing style, with body and mind to understand the designer's creation.
\end{abstract}

\section{Introduction}

Common fashion SHOW music style is varied, such as classical Classc electronic music, Utah Jazz, POP, POP, Techno (Germany and Japan) advanced, psychedelic dance Trance, RAP, RAP, HIP POP, NEW century NEW AGE (quiet, comfortable and carefree music) and so on, there will be a variety of elements or finished product music instruments shrinks mixes again, synthesis of NEW music style, also is what we usually call REMIX, this is common in our fashion SHOW. Both luxury atmosphere, and colorful psychedelic; Elegant and noble, or clever look; These are all can modify clothing can also modify adjectives of music. Let the music to the clothing into the soul, the radiance of runway vivid display. Music use natural sounds as a means of material, but it is not a simple mimic natural sound. Music works must be combined with special subjective feeling to have meaning. To music, fashion show is presented and the concept of the two and the relationship between clothing style analysis. To analyze the structure of music, the organization form is: rhythm, melody, tone. The harmonious elements blend each other mutual penetration is music has a protean performance.

\section{Show the relationship between music and clothes}

Music: rhythm, melody, or harmony voices or instruments of the acoustics of a kind of art. Music and people's life, aesthetic temperament and interest, words, behavior, interpersonal relationship, and so on, have a certain correlation. Fashion show: the model shows in their design clothes, also called 
costume exhibition, is for the promotion of clothes. Designer according to their nature, feeling the life to design various styles of clothes, choose suitable fabric and tailored and customized design, let the model on $\mathrm{T}$ stage along with the music rhythm to perform, thus showing it screation and designers conveys the theme of the concept.

Fashion show leave the music, music is important to the success of a fashion show, its importance lies in the shape space stereo sound effect, build appropriate emotional atmosphere, creating artistic conception and sound environment for performance and appreciation, in fashion shows, music and visual factors such as light, stage visual hearing of a common impression, achieve sound shape color blend, thus build the participation of visual and auditory, daydream space, not only in the full visual feast for the audience the United States, also can capture the latest message to the fashion world. Music can direct the performance of a model, a model more accurately convey the designer's practical philosophy, can also inspire the audience's imagination and understanding of clothing, a better interpretation of the clothing design elements, feel the clothing lasting appeal.

\section{Music form and performance style clothing}

Music and fashion show style is complementary to each other, influence and restrict each other. One aspect, music production and matching is based on the theme of the clothing and design concept are expected on the premise of specific performance style; Another aspect; Music structure also influence to shape of the clothing style positioning and deduce.

The rhythm style changes. Rhythm is all coordination, balance and regular exercise. ।"Rhythm is common of human physiological and psychological needs, is the vitality and the orderly change of mood swings.।" Is mainly refers to music in the music organized sequence, thus appear alternately, on the strength of the regular length. In the fashion show, the rhythm is the music of the ripple, the strength of the regularity in music alternating movement, is the combination point of order, model based on how to adjust the pace of strut walks hair. Generally speaking, the two beat and rhythm is one of the most common on a four count, the rhythm is music conform to the requirements of the model show it is not, as for rhythm depends on different clothing style, due to the clothing style the designer's creation thought and artistic personality, colleagues can also reflect the distinct characteristics of The Times and national characteristics. Interpretations of clothing design concept, therefore, must grasp the good style of positioning. That is to say, clothing style determines the fashion show the rhythm of the music style.

The fashion show the rhythm of the music style and fashion style, the relationship between performance style comparison: rhythm is the beat, the combination of speed and the relationship between stress and so on, it provides the variety of movement forms in the music, both gentle soothing, and there was a magnificent strong; Compact are loose and long and short rhythmic pattern. Changes in the fashion show, the rhythm of the music conveys mood just want to present a concentrated dynamic phenomenon: as the change of rhythm, a model make a rhythmic body movement; B the audience as the metrical model body movement caused by the visual chasing shadows actions; Music rhythm, expresses the inner life of human beings modal, or keyed sad, or happy jumping, or enthusiasm is bold and unrestrained; It is the medium of interpretation clothing concept, it is bridge of model and the audience to achieve emotional communication and resonance.

Here, the lifter fashion show the use of music and all the different rhythm type, a girl spring and summer fashion show music theme series: topic 1 floating youth, 130 take per minute; Topic 2 fresh keeping quiet, every 92; Topic 3 lively and easy, each minute 128; Topic 4 hip hop rock, every 
130-146; Topic 5 personality liberation, every 134-174; Topic 6 cute, 160 take per minute; Finale, every 128. This shows, the change of rhythm style structure determines the performance of the order, the coarse ong fashion show is \"strong start opening - the climax of lively and dynamic expansion performances - weak endl" this structure specifies the clothing shows the change of emotional tone, or quick jump, or slow and uniform, or passionate dash, all affect how the audience's heart rate, breathing, and the corresponding activates internal rhythm. Generally speaking, the pipe quick rhythm is exciting, soothing calm heart leisurely pace.

The connotation of melody . In music, the melody is also called the tune, it is according to a certain height, length and weak relationship between the sound of the line is the main means of music image, is the soul of music. Fashion show music melody dependence, though not as bright as the rhythm, but the melody and rhythm practice most closely elements, gentle rhythm combined for more lyrical melodies, it can boost up the inner peace and dreams. And the combination of the lilting rhythms is jumping more ups and downs of rhythm, it can coruscate gives the passion of youth and vitality. The rhythm of the different style bring diverse melody form enable people to obtain acoustically didn't get to enjoy. The importance of this enough to justify the melody for the fashion show.

Try to chanel Paris spring/summer ready-to-wear fashion show case to analysis the rhythm of the music: due to the clothing style decided to music form and style, so the analysis of the it fashion show music melody before you know chanel this brand clothing design style that helps to understand its music selection. Chanel is a one hundred - year - old French brand, with elegant elegant clothing style is famous for its classic dress. In the 1940 s successful recommend simple and comfortable modern sportswear to women. Formally with casual clothes now as the theme, this show with previous soothing melody reappearance one hundred elegant classic is different, the fashion show the rhythm of the music is the lively, dreamy melody, psychedelic sound to performance of leisure, sports clothing series of contracted, generous, simple but elegant style, the opening to every 138 pat's shop synthetic rock music as a strong, strong drum music, movement, showing the elegance of fusion and leisure fashion style, also shows the spring and summer and vitality to life. The topic of fashion has broken the traditional classic black and white color is given priority to practice, but in blue jeans for carrier formed by a series of apparel design, red and white stripes coat, coral red patent leather high heels, colleagues auxiliary pole with the flavor of the chanel pearl earrings and necklace, bowknot, bags and so on as accessories. The rhythm of the music to the stronger highlights, magnetic singing melody is like concealed in the strong rhythm, no movement, sometimes blurred. In comparison, instrumental music melody is various and rich, sometimes the lyric string, sometimes the strong electric guitar music, and sometimes it is rumbling bass came home, built the ghosts, audio and video world of fantasy. Visible, melody and rhythm is mutual penetration and fusion of each other. But sometimes in the antics of music by unleashing the high-pitched melody like breaking type create psychedelic music background, rebellious, tired. A unique way of explaining the chanel and easy willfulness, leng jun unruly style.

Dominate the music form here, rhythm, melody is the connotation of rhythm expression, for a particular style of clothing and fashion show create the sound environment of appropriate, such forms of music melody in the fashion show music abound. Of course, there are some fashion show music is directly used in a variety of styles of music melody rhythm, such as jazz, rock, rap, tango, hip-hop, etc.

A melody line and clothing design lines corresponding is also worthy of our attention, on the 
visual and auditory collision and fusion of on line, have no stretch of the melody is suitable for display smooth texture, smooth lines, magnificent style of clothing, such as dress, cheongsam; A warm passionate melody suits show unique personality, line is straightforward and singular avant-garde style clothing. The music melody lines and clothing line to realize harmonious and unified, achieve the perfect visual and sound effects.

The performance of the tone color. In music, different instruments live voices will have different characteristics of timbre, tone changes to contrast and formed the music changes, present a colorful world of the hearing. Different instruments in different combinations can produce a variety of complex timbre. With the development of modern electronic technology of electronic music synthesis technology to create a beautiful and colorful music color. Whether Argentina tango bright rhythm; Electronic music or enigma that builds a spatio-temporal crisscross of mysticism; Whether the soul element of style, or jazz leng yan is unusual, or postmodern contracted fatuous... Wonderful voice is always can let fashion concept and style of the fashion show features of perfection. It brings to the fashion show music new miracle, making mitt in spirit's shuttling back and forth in the diversity of the strange sound effects, costume design fashionable element.

If garment shape is within a frame structure, then embodies the colour is the skin. American scholar arne haim thinks: ।"when it comes to facial expression, color was more than form, and the glow of the setting sun and the Mediterranean Sea blue color conveys expression, I'm afraid any can't determine the shape of.I" This, to say the colour is in the form of the key elements in the clothing art, its strong impact on the audience's visual nerve, give a person leave deep impression. Rich color change and its coordination collocation can bring the audience into a fantastic dream world. In fashion show, people for music of auditory between color and visual communication of clothing color, synaesthesia effect on intuition synesthesia, auditory and visual for colour capture form the effective mechanism of be in harmony of interactive communication each other, make both in the light, form, shadow gracious to achieve perfect unity. The sweet, soft, melancholy, had just entered... In this, the color is called dedicating elements directly.

Color is the most important physical characteristics of hue, lightness and purity, it has attracted a certain psychological reaction: feel the changes in temperature, weight, far and near, telescopic, hard and soft feeling impression;

Emotional with nationality and sociality. As for the red, in China, red represents good luck joy; Mexican mayan people think red stands for happiness, wealth and power; The ancient Egyptian think red stands for violence and evil. Therefore, colour also have some culture symbolic significance, different social and ethnic for the different understanding and of color to a great extent, affect the fashion style.

In the fashion show, music style of matching are clothing styles, clothing style positioning and reached the effect of color. As a result, the music style to a certain extent affected by clothing color. Nostalgic retro fashion show can choose baroque or classical violin concerto; The pure and fresh, bright and beautiful of rural style can choose the clarinet or officer of flute playing string works; Urban style of elegant, strong can choose strong disco music or modern rock music; Elegant dress can choose classical symphony; The Chinese nation traditional qipao can choose by pipa, erhu, guzheng national music; Modern leisure sportswear can choose modern vocal music team play.

\section{Conclusion}

Fashion show is a comprehensive art and sex form, it with unique art language to build the dynamic 
deductive fashion and aesthetic - modeling to model human form for display objects, with notes of movement as the modal performance means, so as to build participation of auditory and visual imagination space. Music sound effect can not only make the model performance fully show the design concept and design style of fashion, modern fashion of the era of message; Also can be the audience mind communication and dialogue with the designer.

\section{References}

[1] Yin Min. Clothing for the performing arts Hubei fine arts publishing house. Department of institutions of higher learning costume design professional independence tutorial products from 2008.

[2] took. Model professional sense analysis [J]. Journal of beauty and the age of 2004.

New. [3] BaoMing fashion show art [M]. 2 o05 donghua university press.

[4], hong-li xu GuanZhiKun. Clothing aesthetic tutorial [M]. China's textile press, 2007.

[5] sun. Clothing innovation and practice [M]. Sichuan university press, 2006.

[6] Ding Guyong. Talk about the value of the brand - the relationship of customer and brand [11]. Managers in the world, 2008, 3

[7] taihong lu. Brand value, strengthen brand relationship with the customers [J] world managers, 2006

[8] zhi-min zhou. Based on the theory of brand relationship of brand: research model and prospects [J]. Journal of business economics and management, 2008, 2 (2)

Yong-sheng ma [9], the new development of brand relationship management (CRM) - marketing theory [J] study of finance and economics, 2006, 27 (12)

[10] asiaeyes, xiao-ping Chen. Brand TongJian [M]. Shanghai: Shanghai people's publishing house

[11] xin-ling wang. Brand management strategy [M]. Beijing: economic management publishing house, 2006

[12] He Jia. Brand image planning: the perspective of brand management [sichuan. Shanghai: fudan university press, 2008

[13] to Kevin, kile strategic brand management Beijing: China renmin university press, 1999

[14] David a. g, love rick Cyrus. Joseph M brand leadership [M]. Beijing: xinhua publishing house

[15] David: g A. Brand management law [M]. Inner Mongolia: Inner Mongolia publishing house, 1998 